\title{
Formulation of new correlations in terms of extraterrestrial radiation by optimization of tilt angle for installation of solar photovoltaic systems for maximum power generation: case study of 26 cities in India
}

\author{
AMIT KUMAR YADAV ${ }^{1,2, *}$ and S S CHANDEL ${ }^{1}$ \\ ${ }^{1}$ Centre for Energy and Environmental Engineering, National Institute of Technology, \\ Hamirpur 177005, Himachal Pradesh, India \\ ${ }^{2}$ Electrical and Electronics Engineering Department, National Institute of Technology, Sikkim, Ravangla, \\ Barfung Block, South Sikkim 737139, India \\ e-mail: amit1986.529@rediffmail.com
}

MS received 21 September 2017; revised 1 January 2018; accepted 31 January 2018; published online 18 May 2018

\begin{abstract}
This paper deals with finding of optimum tilt angle for installation of optimized solar photovoltaic system in India. The optimization of tilt angles is performed using measured solar radiation data for 26 cities in India. The tilt angle is changed from $0^{\circ}$ to $90^{\circ}$ at step of $1^{\circ}$ to find out optimum tilt angle for which monthly average solar radiation is maximum. It was found that the optimum tilt angle varies between $0^{\circ}$ and $63^{\circ}$ throughout the year in India. The monthly optimum tilt angle is maximum in December for all cities in India. It is found that increase in maximum solar radiation at monthly optimum tilt angle in comparison to latitude based tilt angle and annual optimum tilt angle varies from $5.85 \%$ to $8.08 \%$ and $5.95 \%$ to $8.34 \%$, respectively, showing monthly optimum tilt angle is beneficial for maximum power generation for different cities in India. The novelty of this study is new correlations of optimum tilt angle in terms of extraterrestrial radiation are developed for estimating monthly optimum tilt angle for installation of photovoltaic systems at different sites in India. The root mean square error in correlation equations varies from 0.1256 to 0.9771 .
\end{abstract}

Keywords. Solar photovoltaic system; optimum tilt angle; correlations.

\section{Introduction}

India has huge solar potential due to its location between the tropic of cancer and the equator. The mean daily global solar radiation of $5 \mathrm{kWh} / \mathrm{m}^{2} /$ day is incident on $58 \%$ part of India showing good option for solar energy applications [1]. The Indian government has taken a major initiative to promote solar energy in the country by launching Jawaharlal Nehru National Solar Mission (JNNSM) in 2010 with a target of 175000 MW by 2022. The objective of National Solar Mission is to establish India as a global leader in solar energy [2]. The solar installed capacity up to 31 March 2017 is $12,288.83 \mathrm{MW}$ and annual growth is $81.71 \%$ in India. The solar energy is utilized through standalone photovoltaic (SAPV) system which converts solar radiation (SR) directly into electricity. These systems are one of the best options for urban, rural and remote locations, including rooftop and building integrated applications. The SR is a function of days, site and time. Therefore to capture maximum SR for maximum power, SAPV system should be

*For correspondence installed at optimum tilt angle so that it faces the sun at all times. The optimum tilt angles (OPTA) are found by searching the angles from $0^{\circ}$ to $90^{\circ}$ for which the total incident radiation on the PV surface is maximum for the period under consideration [3].

The power generated from the SAPV system in India faces many difficulties such as non-availability of measured SR at different locations and optimum sizing i.e., system cost $[4,5]$. Estimation of monthly incident SR on tilted surface is necessary for maximum power generation. The monthly average daily SR data (Indian cities) incident on horizontal surface is available in literature [6, 7]. However very few study related to radiation on tilted surface is reported. Thakur and Chandel [8] calculated optimum tilt angle and studied its effect on energy yield of $190 \mathrm{kWp}$ grid-interactive solar power plant installed at KhatkarKalan in India. The energy yield at yearly, seasonally and monthly optimum tilt angle are found to be $25 \%, 28 \%$, and $29 \%$ more in comparison to fixed tilt $\left(25^{\circ}\right)$ angle of the plant. Yadav and Chandel [9] analyzed different diffuse SR model for calculation of OPTA for Hamirpur, Himachal Pradesh (H.P.) and found that Liu and Jordan model give 
better result with $4.512 \%$ error between calculated and measured optimum tilt angle. Yadav et al [10] calculated OPTA and developed correlations of annual optimum tilt angle as a function of latitude for 15 cities of H.P. using predicted value of SR and found that OPTA varies from $0^{\circ}$ to $65^{\circ}$ for H.P cities. Agarwal et al [11] calculated tilt angle for Delhi and Nandha in Haryana using Liu and Jorden, Reindel, Hay and Badescu models. The optimum tilt angles are $28.29^{\circ}, 29.41^{\circ}, 29.08^{\circ}$ and $27.58^{\circ}$ for Delhi and it is $30.61^{\circ}, 31.66^{\circ}, 31.20^{\circ}$ and $29.58^{\circ}$ for Nandha.

The objective of this study is to determine OPTA for installation of solar photovoltaic system in 26 different cities in India for maximizing incident SR and to obtain new correlations for estimating the OPTA that are useful for industry.

The paper is organized as follows: literature survey overview is given in section 2 and methodology is presented in section 3 . The results and discussion are given in section 4 and conclusions are provided in section 5 .

\section{An overview of literature survey for OPTA calculation}

A number of studies for OPTA calculation for various applications around the world have been presented. However, studies carried out in India are few in number for different sites and the general correlation giving OPTA for India are not obtained.

\subsection{OPTA calculation for maximize solar radiation}

The OPTA are found by searching the angles for which the total radiation incident on the PV surface is maximum for the period under consideration. Ulgen [12] computed OPTA for Izmir, Turkey as summer $4.3^{\circ}$, winter $55.7^{\circ}$ and yearly $30.3^{\circ}$. The energy collected at monthly and yearly tilt angle are 6747.17 and $639 \mathrm{MJ} / \mathrm{m}^{2} /$ year. Talebizadeh et al [13] used Genetic Algorithm for calculating hourly, daily, monthly, seasonally and yearly OPTA in Iran. The gain in SR on seasonally, monthly and daily OPTA are 5.13\%, 6.05\% and $6.89 \%$, showing a gain at monthly and seasonal are almost same. In addition, the energy gain decreases by ignoring ground reflectivity. Pour et al [14] calculated minimum and maximum OPTA as $0.15^{\circ}$ and $57.74^{\circ}$ using the isotropic Liu model for Isfahan. The SR gain varies from $0.04 \%$ to $62 \%$ at a monthly OPTA. Kacira et al [15] determined monthly OPTA of $13^{\circ}$ in June and $61^{\circ}$ in December for Sanliurfa, Turkey. The PV panel mounted on a monthly tilt angle, obtained SR of $1.1 \%$ and $3.9 \%$ with respect to seasonal and latitude based tilt angle. Due to two-axis tracking system the gain in SR is $29.3 \%$, resulting in $34.6 \%$ gain in power generation. Hartley et al [16] utilized Hays model for analyzing hourly deviation in OPTA for Spain. It is found that monthly average SR data is more accurate in obtaining OPTA in comparison to hourly SR data. Mehleri et al [17] tested different types of diffuse models for OPTA formulation and found that "Liu and Jordan" model is accurate. The results demonstrate that changing the tilt angle in cold and hot months enhance generated power reliability. Siraki and Pillay [18] developed modified HDKR (Hay, Davies, Klucher, Reindl) model which consider building shading for calculating the OPTA in urban areas. For a similar clearness index the OPTA is close to latitude for small altitude and it is smaller than latitude for high altitude. Chang [19] used Particle Swarm Optimization technique for calculating the OPTA in Taiwan. The suggested tilt angle is Taipei area $18.16^{\circ}$, Taichung $17.3^{\circ}$, Tainan $16.15^{\circ}$, Kaosiung $15.79^{\circ}$, Hengchung $15.17^{\circ}$, Hualian $17.16^{\circ}$ and Taitung $15.94^{\circ}$. The PV module temperature is high in Kaohsiung in comparison with Hengchun therefore the annual efficiency of a PV module is high in Hengchun. Chatterjee and Keyhani [20] utilized input parameters as latitude, ground reflectivity and 12 months irradiance for estimating OPTA and maximum solar radiation. The difference of $3^{\circ}$ is observed between estimated and calculated OPTA, showing close estimation. This method can be used for estimation of energy output from PV station. Khorasanizadeh et al [21] used anisotropic model to determine OPTA of south-facing solar collectors and solar panels in Tabass city situated in sunny region of Iran. The monthly OPTA vary from $0^{\circ}$ in June and July to $64^{\circ}$ in December. Bakirci [22] developed correlations of OPTA in terms of declination angles for Turkey and correlation coefficients are found to be greater than $99 \%$. The correlations are as follows.

$$
\begin{gathered}
\beta_{o p t}=32.619-1.3629 \delta \\
\beta_{o p t}=34.784-1.3621 \delta-0.0081(\delta)^{2} \\
\beta_{o p t}=34.783-1.4317 \delta-0.0081(\delta)^{2}+0.0002(\delta)^{3}
\end{gathered}
$$

The SR on SAPV system is maximized by OPTA and tracking system, resulting in maximum power generation. Abdallah [23] investigated the effect of two axes, one axis vertical, one axis east-west and one axis north-south tracking system on electrical characteristics of flat plate PV system. The gain in electrical power is increased by $43.87 \%, 37.53 \%, 34.43 \%$ and $15.69 \%$ for the two axes, east-west, vertical and north-south tracking respectively in comparison to fixed tilt angle of $32^{\circ}$ for Amman, Jordan.

Beringer et al [24] investigated the performance of solar cell at OPTA in Germany. It is found that tilt angle effect on performance is minimum due to temperature effects. Bojić et al [25] used Hooke-Jeeves algorithm for computing OPTA at which electrical energy generation is maximized. Chang [26] used sequential neural-network approximation and orthogonal arrays (SNAOA) to estimate the OPTA for maximum output power energy of PV modules in seven regions of Taiwan. The annual OPTA is $23.251^{\circ}$ for Taipei site. 
Table 1. Latitude based OPTA correlations.

\begin{tabular}{|c|c|c|}
\hline Study & Correlation of OPTA & Application \\
\hline Heywood [28] & $\phi-10^{\circ}$ & The minus sign refers to the summer season \\
\hline Lunde [29] & $\phi \pm 15^{\circ}$ & and the positive sign is for the winter \\
\hline Chinnery [30] & $\phi+10^{\circ}$ & \\
\hline Kern and Harris [31] & $\phi+10^{\circ}$ & \\
\hline Yellot [32] & $\phi+20^{\circ}$ & \\
\hline Elminir et al [33] & $\left(\phi+15^{\circ}\right) \pm 15^{\circ}$ & \\
\hline Tang and $\mathrm{Wu}[34]$ & $\phi-3^{\circ}, \phi+4^{\circ}, \phi-5^{\circ}, \phi-10^{\circ}$ & \\
\hline Talebizadeh et al [35] & $\begin{array}{c}1.073 \phi+10.3 \text { (winter), } 0.48850 \phi-10.27 \text { (spring) } \\
0.2637 \phi+4.961 \text { (summer), } 0.8966 \phi+23.81 \text { (autumn) } \\
0.6804 \phi+7.203 \text { (yearly) }\end{array}$ & \\
\hline
\end{tabular}

Roux [27] performed study on solar tracker for locations in South Africa and suggested that for solar collector a fixed tilt angle of $30^{\circ}$ collects $98 \%$ of maximum SR. The plots of contour are presented to show the effect of fixed tilt and azimuth angle on maximum SR.

Several authors have developed correlations of OPTA in terms of latitude $(\phi)$ as shown in table 1 .

Moreover, in a comprehensive review by Yadav and Chandel [36], the OPTA calculation has been identified as an important research area for maximum power generation by maximizing SR.

\section{Methodology}

\subsection{Optimum tilt angle determination}

The extraterrestrial radiation is the SR received outside earth's surface and its intensity varies throughout the year. The average value of extraterrestrial radiation i.e., the solar constant is $S_{0}=1367 \mathrm{~W} / \mathrm{m}^{2}$.

The polar axis of earth is inclined at an angle of $66.55^{\circ}$ to the elliptical plane and by $23.45^{\circ}$ from perpendicular to elliptical plane. The rotation of the earth on its inclined polar axis with respect to elliptic plane is responsible for different seasons on earth. It causes lengthy days in summer and shorter days in winter. The angle made by the lines joining the centre of the earth to centre of the sun with its projection on the equatorial plane of the earth is called the declination angle. It varies due to the inclination of the earth's polar axis and its revolution around the sun. It varies between $-23.45^{\circ}$ and $23.45^{\circ}$.

The angular displacement of the sun about east or west of local meridian due to the rotary motion of earth on axis is called hour angle $(w)$. It is an expression explaining the variation between local solar time and solar noon. After solar noon the hour angle measures time in duration of $1^{\circ}$ for each 4 minutes or $15^{\circ}$ per hour. The hour angle is positive after solar noon and it is negative before solar noon.

The incident radiation reaches the earth's surface without being absorbed or scattered, is called beam radiation. Some of the radiation from the sun is scattered back to the atmosphere and a part of it is scattered to earth, this scattered radiation reaching the earth's surface is called diffuse radiation $\left(H_{d}\right)$.

When the SR reaches the earth's surface, some of it is reflected by the ground and other objects on the ground. This radiation is called reflected radiation $\left(H_{r}\right)$. The total SR on a horizontal surface is called global radiation $\left(H_{g}\right)$ which is the sum of the beam, diffused and reflected radiations. For maximum utilization of SR on PV panel the tilt angle $(\beta)$ is used which lies between $0^{\circ}$ and $90^{\circ}$ [37]. The angle between the plane of the PV panel surface and horizontal is called tilt angle $(\beta)$ is shown in figure 1 . The optimum tilt angle for maximizing incident $\mathrm{SR}\left(\beta_{\text {opt }}\right)$ is calculated by changing $\beta$ between $0^{\circ}$ to $90^{\circ}$ at step of $1^{\circ}$ for which $H_{T}$ is maximum as shown in figure 2 [38].

\section{Results and discussion}

The monthly OPTA $\left(\beta_{\text {opt }}\right)$ are calculated using developed program in MATLAB (R2011a) based on flow chart. This program utilizes monthly average SR (table 2) and for obtaining monthly OPTA, $\beta$ is changed from $0^{\circ}$ to $90^{\circ}$ at an

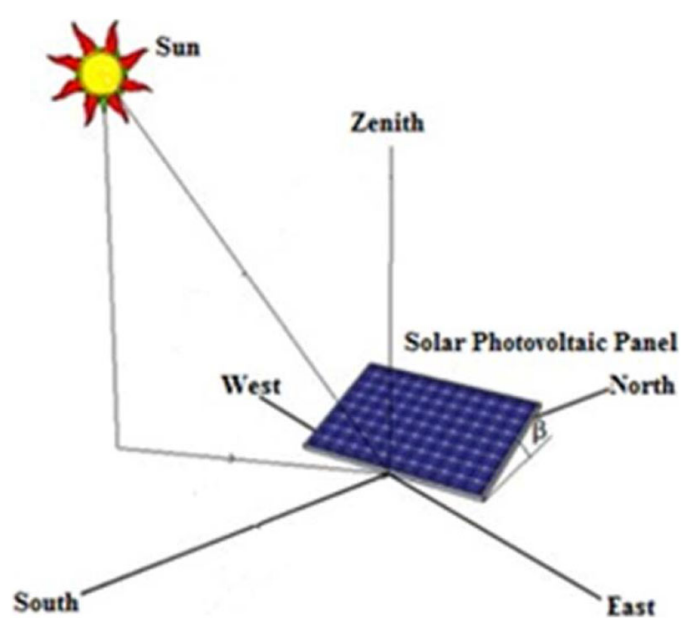

Figure 1. PV panel installed at a tilt angle. 


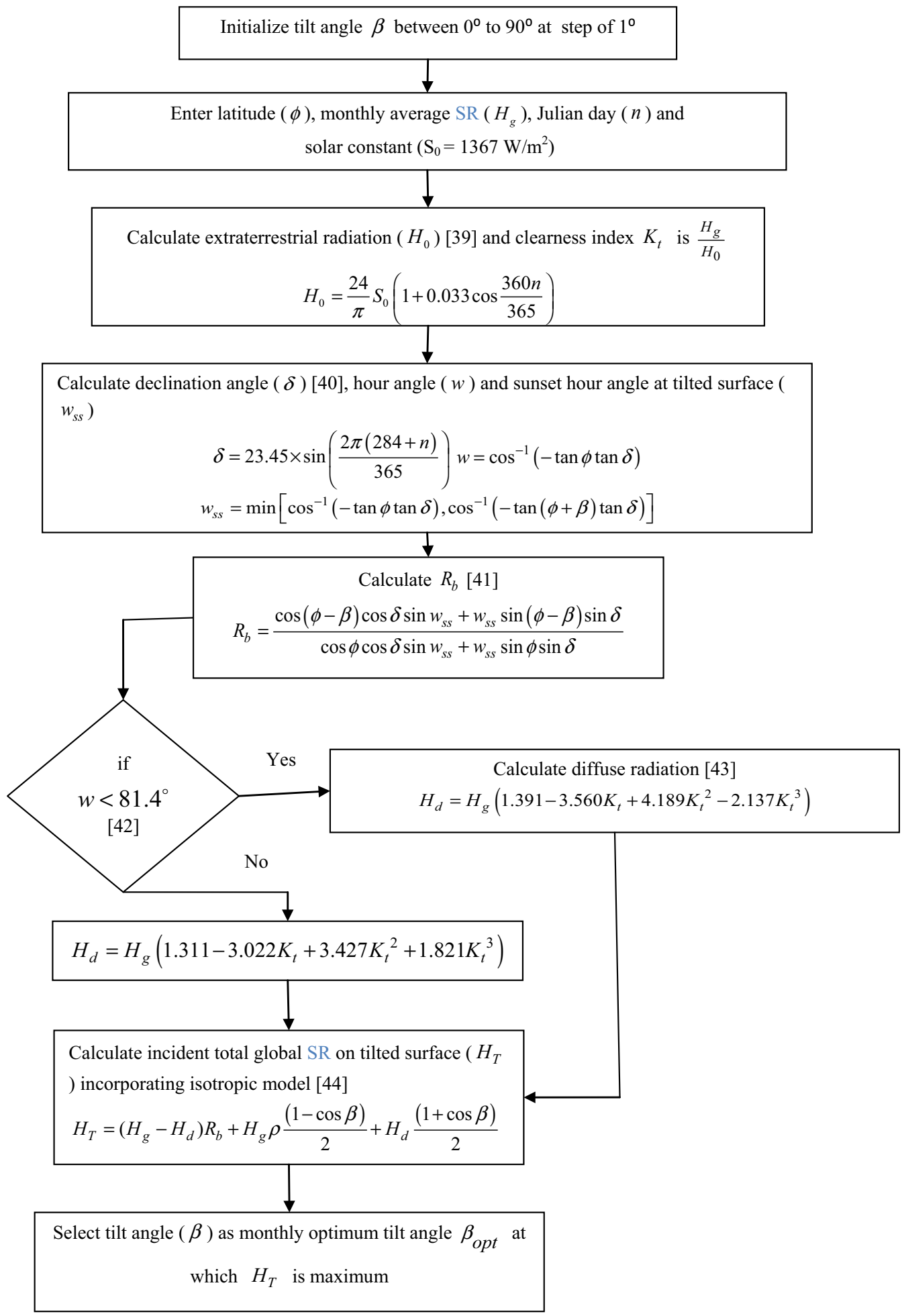

Figure 2. Flow chart for OPTA calculation.

interval of $1^{\circ}$. The tilt angle $\beta$ which cause the maximum value of $H_{T}$ is selected by the program as the optimum tilt angle $\beta_{\text {opt }}$ for that month (table 2).

It was found that the OPTA changed between $0^{\circ}$ (April, May, June, July and August) and $41^{\circ}$ (December) for Minicoy, $0^{\circ}$ (April, May, June, July and August) and $42^{\circ}$
(December) for Thiruvanathpuram, $0^{\circ}$ (April, May, June, July and August) and $44^{\circ}$ (December) for Port Blair, $0^{\circ}$ (April, May, June, July and August) and $45^{\circ}$ (December) for Bangalore, $0^{\circ}$ (April, May, June, July and August) and $45^{\circ}$ (December) for Chennai, $0^{\circ}$ (April, May, June, July and August) and $48^{\circ}$ (December) for Panjim, $0^{\circ}$ (April, May, 
Table 2. Monthly average daily global (measured), diffuse (calculated) and extraterrestrial SR on a horizontal surface, clearness index, optimum tilt-angle and monthly average daily global radiation on optimum tilted surface for 26 cities of India.

\begin{tabular}{|c|c|c|c|c|c|c|c|c|c|}
\hline Cities & Months & $H_{g}$ & $H_{d}$ & $H_{0}$ & $K_{t}$ & $\beta_{o p t}$ & $H_{T}$ & $\mathrm{HL}$ & HY \\
\hline \multirow[t]{13}{*}{ Minicoy } & Jan. & 4.93 & 0.92 & 9.48 & 0.52 & 39 & 6.05 & 5.35 & 5.55 \\
\hline & Feb. & 5.61 & 1.04 & 9.82 & 0.57 & 28 & 6.21 & 5.91 & 6.04 \\
\hline & Mar. & 6.05 & 1.07 & 10.37 & 0.58 & 12 & 6.16 & 6.15 & 6.16 \\
\hline & Apr. & 5.93 & 1.13 & 9.85 & 0.60 & 0 & 5.93 & 5.81 & 5.69 \\
\hline & May & 5.05 & 0.90 & 9.88 & 0.51 & 0 & 5.05 & 4.79 & 4.60 \\
\hline & Jun. & 4.44 & 0.85 & 9.21 & 0.48 & 0 & 4.44 & 4.15 & 3.95 \\
\hline & Jul. & 4.58 & 0.82 & 9.89 & 0.46 & 0 & 4.58 & 4.30 & 4.11 \\
\hline & Aug. & 4.88 & 0.85 & 10.12 & 0.48 & 0 & 4.88 & 4.71 & 4.58 \\
\hline & Sep. & 5.09 & 0.90 & 9.98 & 0.50 & 5 & 5.10 & 5.09 & 5.06 \\
\hline & Oct. & 5.003 & 0.86 & 10.27 & 0.48 & 23 & 5.36 & 5.21 & 5.29 \\
\hline & Nov. & 4.63 & 0.82 & 10.04 & 0.46 & 36 & 5.53 & 4.99 & 5.16 \\
\hline & Dec. & 4.6 & 0.83 & 9.80 & 0.47 & 41 & 5.84 & 5.04 & 5.25 \\
\hline & Average & 5.07 & 0.92 & 9.89 & 0.51 & 13.18 & 5.43 & 5.12 & 5.12 \\
\hline \multirow[t]{13}{*}{ Thiruvanathpuram } & Jan. & 5.53 & 1.07 & 9.47 & 0.58 & 39 & 6.78 & 6.01 & 6.23 \\
\hline & Feb. & 6.12 & 1.20 & 9.82 & 0.62 & 28 & 6.77 & 6.45 & 6.59 \\
\hline & Mar. & 6.5 & 1.21 & 10.36 & 0.62 & 12 & 6.62 & 6.61 & 6.62 \\
\hline & Apr. & 5.93 & 1.13 & 9.85 & 0.60 & 0 & 5.93 & 5.80 & 5.69 \\
\hline & May & 5.44 & 0.99 & 9.88 & 0.55 & 0 & 5.44 & 5.15 & 4.96 \\
\hline & Jun. & 4.82 & 0.93 & 9.21 & 0.52 & 0 & 4.82 & 4.50 & 4.29 \\
\hline & Jul. & 4.95 & 0.88 & 9.89 & 0.50 & 0 & 4.95 & 4.65 & 4.45 \\
\hline & Aug. & 5.27 & 0.92 & 10.12 & 0.52 & 0 & 5.27 & 5.09 & 4.94 \\
\hline & Sep. & 5.7 & 1.04 & 9.97 & 0.57 & 5 & 5.72 & 5.71 & 5.67 \\
\hline & Oct. & 5.04 & 0.86 & 10.26 & 0.49 & 23 & 5.41 & 5.26 & 5.34 \\
\hline & Nov. & 4.6 & 0.82 & 10.03 & 0.45 & 36 & 5.50 & 4.96 & 5.13 \\
\hline & Dec. & 5.01 & 0.90 & 9.79 & 0.51 & 42 & 6.38 & 5.50 & 5.73 \\
\hline & Average & 5.41 & 0.99 & 9.89 & 0.54 & 13.27 & 5.80 & 5.47 & 5.47 \\
\hline \multirow[t]{13}{*}{ Port Blair } & Jan. & 5.12 & 0.98 & 9.37 & 0.54 & 42 & 6.50 & 5.77 & 5.92 \\
\hline & Feb. & 5.85 & 1.13 & 9.72 & 0.60 & 31 & 6.63 & 6.32 & 6.41 \\
\hline & Mar. & 5.89 & 1.04 & 10.26 & 0.57 & 16 & 6.07 & 6.06 & 6.07 \\
\hline & Apr. & 5.76 & 1.09 & 9.75 & 0.59 & 0 & 5.76 & 5.61 & 5.54 \\
\hline & May & 4.37 & 0.80 & 9.77 & 0.44 & 0 & 4.37 & 4.08 & 3.98 \\
\hline & Jun. & 3.87 & 0.78 & 9.10 & 0.42 & 0 & 3.87 & 3.54 & 3.43 \\
\hline & Jul. & 3.82 & 0.76 & 9.78 & 0.39 & 0 & 3.82 & 3.53 & 3.43 \\
\hline & Aug. & 4.02 & 0.76 & 10.01 & 0.40 & 0 & 4.02 & 3.85 & 3.78 \\
\hline & Sep. & 4.3 & 0.79 & 9.88 & 0.43 & 8 & 4.33 & 4.32 & 4.31 \\
\hline & Oct. & 4.48 & 0.80 & 10.16 & 0.44 & 26 & 4.90 & 4.77 & 4.82 \\
\hline & Nov. & 4.65 & 0.83 & 9.93 & 0.46 & 39 & 5.74 & 5.19 & 5.31 \\
\hline & Dec. & 4.74 & 0.86 & 9.68 & 0.49 & 44 & 6.26 & 5.42 & 5.58 \\
\hline & Average & 4.74 & 0.89 & 9.78 & 0.48 & 14.9 & 5.19 & 4.87 & 4.88 \\
\hline \multirow[t]{13}{*}{ Bangalore } & Jan. & 5.67 & 1.14 & 9.33 & 0.60 & 42 & 7.25 & 6.45 & 6.60 \\
\hline & Feb. & 6.48 & 1.37 & 9.68 & 0.66 & 32 & 7.38 & 7.05 & 7.14 \\
\hline & Mar. & 6.58 & 1.27 & 10.23 & 0.64 & 16 & 6.80 & 6.79 & 6.80 \\
\hline & Apr. & 6.56 & 1.39 & 9.71 & 0.67 & 0 & 6.56 & 6.39 & 6.32 \\
\hline & May & 6.35 & 1.30 & 9.73 & 0.65 & 0 & 6.35 & 5.91 & 5.78 \\
\hline & Jun. & 4.92 & 0.97 & 9.06 & 0.54 & 0 & 4.92 & 4.47 & 4.35 \\
\hline & Jul. & 4.64 & 0.84 & 9.74 & 0.47 & 0 & 4.64 & 4.26 & 4.15 \\
\hline & Aug. & 4.48 & 0.81 & 9.98 & 0.44 & 0 & 4.48 & 4.28 & 4.21 \\
\hline & Sep. & 5.24 & 0.94 & 9.84 & 0.53 & 9 & 5.30 & 5.29 & 5.27 \\
\hline & Oct. & 5.11 & 0.89 & 10.12 & 0.50 & 28 & 5.64 & 5.48 & 5.54 \\
\hline & Nov. & 4.84 & 0.86 & 9.89 & 0.49 & 40 & 6.05 & 5.47 & 5.58 \\
\hline & Dec. & 4.81 & 0.88 & 9.64 & 0.49 & 45 & 6.44 & 5.57 & 5.72 \\
\hline & Average & 5.47 & 1.05 & 9.75 & 0.56 & 15.45 & 5.98 & 5.62 & 5.62 \\
\hline
\end{tabular}


Table 2 continued

\begin{tabular}{|c|c|c|c|c|c|c|c|c|c|}
\hline Cities & Months & $H_{g}$ & $H_{d}$ & $H_{0}$ & $K_{t}$ & $\beta_{o p t}$ & $H_{T}$ & $\mathrm{HL}$ & HY \\
\hline \multirow[t]{13}{*}{ Chennai } & Jan. & 4.89 & 0.93 & 9.31 & 0.52 & 43 & 6.32 & 5.61 & 5.73 \\
\hline & Feb. & 5.85 & 1.14 & 9.66 & 0.60 & 32 & 6.71 & 6.40 & 6.48 \\
\hline & Mar. & 6.51 & 1.25 & 10.21 & 0.63 & 17 & 6.75 & 6.73 & 6.74 \\
\hline & Apr. & 6.6 & 1.41 & 9.69 & 0.68 & 0 & 6.60 & 6.42 & 6.36 \\
\hline & May & 6.26 & 1.27 & 9.71 & 0.64 & 0 & 6.26 & 5.81 & 5.70 \\
\hline & Jun. & 5.71 & 1.23 & 9.04 & 0.63 & 0 & 5.71 & 5.19 & 5.07 \\
\hline & Jul. & 5.27 & 0.97 & 9.71 & 0.54 & 0 & 5.27 & 4.83 & 4.72 \\
\hline & Aug. & 5.2 & 0.92 & 9.95 & 0.52 & 0 & 5.20 & 4.96 & 4.88 \\
\hline & Sep. & 5.39 & 0.98 & 9.82 & 0.54 & 10 & 5.45 & 5.44 & 5.43 \\
\hline & Oct. & 4.55 & 0.81 & 10.10 & 0.45 & 28 & 5.04 & 4.90 & 4.94 \\
\hline & Nov. & 3.99 & 0.77 & 9.86 & 0.40 & 40 & 4.99 & 4.53 & 4.61 \\
\hline & Dec. & 4.15 & 0.79 & 9.62 & 0.43 & 45 & 5.57 & 4.83 & 4.94 \\
\hline & Average & 5.36 & 1.04 & 9.72 & 0.55 & 15.63 & 5.82 & 5.47 & 5.47 \\
\hline \multirow[t]{13}{*}{ Panjim } & Jan. & 5.52 & 1.12 & 9.19 & 0.60 & 45 & 7.32 & 6.53 & 6.60 \\
\hline & Feb. & 6.22 & 1.29 & 9.55 & 0.65 & 34 & 7.27 & 6.90 & 6.99 \\
\hline & Mar. & 6.54 & 1.28 & 10.10 & 0.64 & 19 & 6.85 & 6.84 & 6.84 \\
\hline & Apr. & 6.72 & 1.50 & 9.59 & 0.70 & 0 & 6.72 & 6.55 & 6.51 \\
\hline & May & 6.56 & 1.43 & 9.59 & 0.68 & 0 & 6.56 & 6.05 & 5.99 \\
\hline & Jun. & 4.63 & 0.91 & 8.92 & 0.51 & 0 & 4.63 & 4.14 & 4.09 \\
\hline & Jul. & 4.1 & 0.78 & 9.59 & 0.42 & 0 & 4.10 & 3.71 & 3.67 \\
\hline & Aug. & 4.4 & 0.80 & 9.84 & 0.44 & 0 & 4.40 & 4.18 & 4.15 \\
\hline & Sep. & 5.38 & 0.99 & 9.72 & 0.55 & 12 & 5.48 & 5.48 & 5.47 \\
\hline & Oct. & 5.42 & 0.97 & 9.99 & 0.54 & 30 & 6.11 & 5.95 & 5.98 \\
\hline & Nov. & 5.32 & 0.97 & 9.74 & 0.54 & 43 & 6.87 & 6.22 & 6.28 \\
\hline & Dec. & 5.16 & 0.97 & 9.49 & 0.54 & 48 & 7.18 & 6.23 & 6.31 \\
\hline & Average & 5.5 & 1.09 & 9.61 & 0.57 & 16.9 & 6.12 & 5.73 & 5.74 \\
\hline \multirow{13}{*}{ Hyderabad } & Jan. & 5.45 & 1.12 & 9.09 & 0.59 & 46 & 7.41 & 6.62 & 6.65 \\
\hline & Feb. & 6.11 & 1.28 & 9.45 & 0.64 & 36 & 7.28 & 6.96 & 6.98 \\
\hline & Mar. & 6.72 & 1.38 & 10.00 & 0.67 & 21 & 7.11 & 7.09 & 7.10 \\
\hline & Apr. & 6.9 & 1.62 & 9.49 & 0.72 & 2 & 6.91 & 6.73 & 6.71 \\
\hline & May & 6.63 & 1.49 & 9.49 & 0.69 & 0 & 6.63 & 6.07 & 6.05 \\
\hline & Jun. & 5.59 & 1.23 & 8.82 & 0.63 & 0 & 5.59 & 4.97 & 4.94 \\
\hline & Jul. & 5.13 & 0.96 & 9.49 & 0.54 & 0 & 5.13 & 4.61 & 4.59 \\
\hline & Aug. & 4.87 & 0.88 & 9.74 & 0.50 & 0 & 4.87 & 4.62 & 4.60 \\
\hline & Sep. & 5.49 & 1.03 & 9.63 & 0.57 & 14 & 5.62 & 5.61 & 5.61 \\
\hline & Oct. & 5.18 & 0.93 & 9.89 & 0.52 & 32 & 5.94 & 5.78 & 5.79 \\
\hline & Nov. & 5.02 & 0.92 & 9.64 & 0.52 & 44 & 6.64 & 6.01 & 6.04 \\
\hline & Dec. & 4.98 & 0.94 & 9.39 & 0.53 & 49 & 7.12 & 6.19 & 6.23 \\
\hline & Average & 5.67 & 1.15 & 9.51 & 0.59 & 18 & 6.35 & 5.94 & 5.94 \\
\hline \multirow[t]{13}{*}{ Vishakapatnam } & Jan. & 4.83 & 0.94 & 9.09 & 0.53 & 47 & 6.61 & 5.89 & 5.92 \\
\hline & Feb. & 5.55 & 1.08 & 9.45 & 0.58 & 37 & 6.65 & 6.34 & 6.36 \\
\hline & Mar. & 6.06 & 1.14 & 10.00 & 0.60 & 21 & 6.42 & 6.40 & 6.41 \\
\hline & Apr. & 6.38 & 1.38 & 9.49 & 0.67 & 2 & 6.39 & 6.22 & 6.20 \\
\hline & May & 6.16 & 1.29 & 9.48 & 0.64 & 0 & 6.16 & 5.63 & 5.61 \\
\hline & Jun. & 4.85 & 0.99 & 8.82 & 0.55 & 0 & 4.85 & 4.31 & 4.28 \\
\hline & Jul. & 4.45 & 0.83 & 9.48 & 0.46 & 0 & 4.45 & 3.99 & 3.97 \\
\hline & Aug. & 4.54 & 0.83 & 9.74 & 0.46 & 0 & 4.54 & 4.30 & 4.28 \\
\hline & Sep. & 4.73 & 0.87 & 9.62 & 0.49 & 14 & 4.85 & 4.85 & 4.84 \\
\hline & Oct. & 4.89 & 0.87 & 9.89 & 0.49 & 32 & 5.61 & 5.45 & 5.47 \\
\hline & Nov. & 4.55 & 0.84 & 9.64 & 0.47 & 44 & 6.03 & 5.46 & 5.49 \\
\hline & Dec. & 4.53 & 0.85 & 9.38 & 0.48 & 49 & 6.48 & 5.63 & 5.67 \\
\hline & Average & 5.13 & 0.99 & 9.51 & 0.53 & 18.09 & 5.75 & 5.37 & 5.37 \\
\hline
\end{tabular}


Table 2 continued

\begin{tabular}{|c|c|c|c|c|c|c|c|c|c|}
\hline Cities & Months & $H_{g}$ & $H_{d}$ & $H_{0}$ & $K_{t}$ & $\beta_{o p t}$ & $H_{T}$ & $\mathrm{HL}$ & HY \\
\hline \multirow[t]{13}{*}{ Pune } & Jan. & 4.8 & 0.94 & 9.02 & 0.53 & 48 & 6.66 & 5.94 & 5.95 \\
\hline & Feb. & 5.71 & 1.15 & 9.39 & 0.60 & 38 & 6.90 & 6.59 & 6.60 \\
\hline & Mar. & 6.41 & 1.27 & 9.93 & 0.64 & 22 & 6.83 & 6.82 & 6.82 \\
\hline & Apr. & 6.8 & 1.59 & 9.43 & 0.72 & 3 & 6.81 & 6.63 & 6.62 \\
\hline & May & 6.99 & 1.70 & 9.42 & 0.74 & 0 & 6.99 & 6.40 & 6.39 \\
\hline & Jun. & 5.36 & 1.16 & 8.75 & 0.61 & 0 & 5.36 & 4.74 & 4.74 \\
\hline & Jul. & 4.47 & 0.84 & 9.41 & 0.47 & 0 & 4.47 & 4.00 & 3.99 \\
\hline & Aug. & 4.35 & 0.81 & 9.67 & 0.45 & 0 & 4.35 & 4.12 & 4.11 \\
\hline & Sep. & 5.2 & 0.97 & 9.56 & 0.54 & 15 & 5.35 & 5.34 & 5.34 \\
\hline & Oct. & 5.34 & 0.97 & 9.83 & 0.54 & 33 & 6.18 & 6.02 & 6.02 \\
\hline & Nov. & 4.9 & 0.90 & 9.57 & 0.51 & 45 & 6.58 & 5.97 & 5.98 \\
\hline & Dec. & 4.57 & 0.86 & 9.31 & 0.49 & 50 & 6.64 & 5.78 & 5.79 \\
\hline & Average & 5.41 & 1.10 & 9.44 & 0.57 & 18.72 & 6.09 & 5.69 & 5.70 \\
\hline \multirow[t]{13}{*}{ Mumbai } & Jan. & 4.6 & 0.90 & 8.99 & 0.51 & 48 & 6.44 & 5.74 & 5.75 \\
\hline & Feb. & 5.41 & 1.06 & 9.36 & 0.57 & 38 & 6.59 & 6.28 & 6.29 \\
\hline & Mar. & 6.17 & 1.20 & 9.90 & 0.62 & 23 & 6.60 & 6.59 & 6.59 \\
\hline & Apr. & 6.61 & 1.51 & 9.40 & 0.70 & 4 & 6.62 & 6.45 & 6.44 \\
\hline & May & 6.48 & 1.46 & 9.38 & 0.69 & 0 & 6.48 & 5.92 & 5.91 \\
\hline & Jun. & 4.85 & 1.00 & 8.72 & 0.55 & 0 & 4.85 & 4.28 & 4.27 \\
\hline & Jul. & 3.73 & 0.76 & 9.38 & 0.39 & 0 & 3.73 & 3.34 & 3.33 \\
\hline & Aug. & 4.03 & 0.77 & 9.64 & 0.41 & 0 & 4.03 & 3.81 & 3.81 \\
\hline & Sep. & 4.54 & 0.84 & 9.53 & 0.47 & 16 & 4.68 & 4.67 & 4.67 \\
\hline & Oct. & 5.003 & 0.90 & 9.79 & 0.51 & 34 & 5.82 & 5.66 & 5.66 \\
\hline & Nov. & 4.61 & 0.85 & 9.53 & 0.48 & 46 & 6.24 & 5.66 & 5.67 \\
\hline & Dec. & 4.29 & 0.82 & 9.27 & 0.46 & 51 & 6.28 & 5.48 & 5.49 \\
\hline & Average & 5.03 & 1.01 & 9.41 & 0.53 & 19.27 & 5.70 & 5.32 & 5.32 \\
\hline \multirow[t]{13}{*}{ Nagpur } & Jan. & 4.48 & 0.89 & 8.85 & 0.50 & 50 & 6.46 & 5.77 & 5.75 \\
\hline & Feb. & 5.33 & 1.06 & 9.23 & 0.57 & 40 & 6.64 & 6.33 & 6.32 \\
\hline & Mar. & 6.09 & 1.19 & 9.78 & 0.62 & 25 & 6.59 & 6.58 & 6.57 \\
\hline & Apr. & 6.65 & 1.57 & 9.27 & 0.71 & 6 & 6.68 & 6.50 & 6.51 \\
\hline & May & 6.55 & 1.53 & 9.25 & 0.70 & 0 & 6.55 & 5.95 & 5.97 \\
\hline & Jun. & 5.23 & 1.15 & 8.58 & 0.60 & 0 & 5.23 & 4.58 & 4.60 \\
\hline & Jul. & 4.11 & 0.80 & 9.24 & 0.44 & 0 & 4.11 & 3.65 & 3.66 \\
\hline & Aug. & 4.1 & 0.79 & 9.51 & 0.43 & 0 & 4.10 & 3.88 & 3.89 \\
\hline & Sep. & 4.87 & 0.91 & 9.41 & 0.51 & 18 & 5.06 & 5.06 & 5.06 \\
\hline & Oct. & 5.18 & 0.95 & 9.66 & 0.53 & 36 & 6.14 & 5.98 & 5.97 \\
\hline & Nov. & 4.54 & 0.85 & 9.39 & 0.48 & 48 & 6.32 & 5.74 & 5.72 \\
\hline & Dec. & 4.27 & 0.42 & 9.13 & 0.46 & 54 & 6.80 & 5.82 & 5.79 \\
\hline & Average & 5.12 & 1.01 & 9.28 & 0.55 & 20.63 & 5.89 & 5.49 & 5.48 \\
\hline \multirow[t]{13}{*}{ Bhavnagar } & Jan. & 4.97 & 0.33 & 8.80 & 0.56 & 53 & 7.76 & 6.76 & 6.69 \\
\hline & Feb. & 5.81 & 1.23 & 9.18 & 0.63 & 40 & 7.25 & 6.93 & 6.90 \\
\hline & Mar. & 6.71 & 1.45 & 9.73 & 0.68 & 25 & 7.27 & 7.26 & 7.25 \\
\hline & Apr. & 7.28 & 1.95 & 9.23 & 0.78 & 6 & 7.32 & 7.12 & 7.15 \\
\hline & May & 7.37 & 2.02 & 9.20 & 0.80 & 0 & 7.37 & 6.71 & 6.76 \\
\hline & Jun. & 6.19 & 1.61 & 8.53 & 0.72 & 0 & 6.19 & 5.44 & 5.49 \\
\hline & Jul. & 4.52 & 0.87 & 9.19 & 0.49 & 0 & 4.52 & 4.00 & 4.03 \\
\hline & Aug. & 4.48 & 0.84 & 9.46 & 0.47 & 0 & 4.48 & 4.24 & 4.26 \\
\hline & Sep. & 5.53 & 1.09 & 9.37 & 0.59 & 18 & 5.76 & 5.75 & 5.76 \\
\hline & Oct. & 5.85 & 1.14 & 9.62 & 0.60 & 36 & 6.96 & 6.78 & 6.75 \\
\hline & Nov. & 5.09 & 0.97 & 9.34 & 0.54 & 48 & 7.15 & 6.50 & 6.45 \\
\hline & Dec. & 4.59 & 0.38 & 9.08 & 0.50 & 55 & 7.48 & 6.38 & 6.31 \\
\hline & Average & 5.7 & 1.16 & 9.23 & 0.61 & 20.72 & 6.62 & 6.16 & 6.15 \\
\hline
\end{tabular}


Table 2 continued

\begin{tabular}{|c|c|c|c|c|c|c|c|c|c|}
\hline Cities & Months & $H_{g}$ & $H_{d}$ & $H_{0}$ & $K_{t}$ & $\beta_{o p t}$ & $H_{T}$ & $\mathrm{HL}$ & HY \\
\hline \multirow[t]{13}{*}{ Kolkatta } & Jan. & 3.75 & 0.46 & 8.76 & 0.42 & 53 & 5.75 & 5.07 & 5.04 \\
\hline & Feb. & 4.35 & 0.84 & 9.14 & 0.47 & 42 & 5.51 & 5.26 & 5.24 \\
\hline & Mar. & 5.27 & 0.97 & 9.69 & 0.54 & 27 & 5.76 & 5.75 & 5.75 \\
\hline & Apr. & 5.85 & 1.24 & 9.19 & 0.63 & 8 & 5.88 & 5.73 & 5.74 \\
\hline & May & 5.73 & 1.20 & 9.15 & 0.62 & 0 & 5.73 & 5.18 & 5.20 \\
\hline & Jun. & 4.76 & 1.01 & 8.49 & 0.56 & 0 & 4.76 & 4.15 & 4.17 \\
\hline & Jul. & 4.19 & 0.82 & 9.14 & 0.45 & 0 & 4.19 & 3.70 & 3.72 \\
\hline & Aug. & 4.32 & 0.82 & 9.42 & 0.45 & 2 & 4.32 & 4.09 & 4.10 \\
\hline & Sep. & 4.13 & 0.80 & 9.32 & 0.44 & 20 & 4.32 & 4.32 & 4.32 \\
\hline & Oct. & 4.24 & 0.80 & 9.57 & 0.44 & 37 & 5.09 & 4.95 & 4.94 \\
\hline & Nov. & 3.84 & 0.77 & 9.30 & 0.41 & 49 & 5.42 & 4.94 & 4.92 \\
\hline & Dec. & 3.52 & 0.51 & 9.03 & 0.38 & 54 & 5.60 & 4.84 & 4.81 \\
\hline & Average & 4.5 & 0.85 & 9.188 & 0.49 & 21.72 & 5.20 & 4.83 & 4.83 \\
\hline \multirow[t]{13}{*}{ Ahmedabad } & Jan. & 4.53 & 0.37 & 8.71 & 0.52 & 53 & 7.19 & 6.28 & 6.25 \\
\hline & Feb. & 5.43 & 1.11 & 9.09 & 0.59 & 43 & 6.91 & 6.60 & 6.58 \\
\hline & Mar. & 6.34 & 1.32 & 9.64 & 0.65 & 28 & 6.94 & 6.93 & 6.93 \\
\hline & Apr. & 6.95 & 1.79 & 9.14 & 0.76 & 9 & 7.00 & 6.81 & 6.82 \\
\hline & May & 6.99 & 1.83 & 9.10 & 0.76 & 0 & 6.99 & 6.34 & 6.36 \\
\hline & Jun. & 6.01 & 1.55 & 8.44 & 0.71 & 0 & 6.01 & 5.26 & 5.28 \\
\hline & Jul. & 4.31 & 0.84 & 9.09 & 0.47 & 0 & 4.31 & 3.80 & 3.82 \\
\hline & Aug. & 4.3 & 0.82 & 9.37 & 0.45 & 2 & 4.30 & 4.07 & 4.08 \\
\hline & Sep. & 5.17 & 1.00 & 9.28 & 0.55 & 21 & 5.43 & 5.42 & 5.42 \\
\hline & Oct. & 5.25 & 0.99 & 9.53 & 0.55 & 38 & 6.35 & 6.18 & 6.17 \\
\hline & Nov. & 4.65 & 0.88 & 9.25 & 0.50 & 50 & 6.66 & 6.06 & 6.04 \\
\hline & Dec. & 4.23 & 0.42 & 8.98 & 0.47 & 57 & 6.99 & 5.99 & 5.96 \\
\hline & Average & 5.35 & 1.08 & 9.13 & 0.58 & 22.54 & 6.26 & 5.81 & 5.81 \\
\hline \multirow{13}{*}{ Bhopal } & Jan. & 4.38 & 0.38 & 8.69 & 0.50 & 54 & 6.95 & 6.09 & 6.02 \\
\hline & Feb. & 5.2 & 1.04 & 9.08 & 0.57 & 42 & 6.63 & 6.34 & 6.30 \\
\hline & Mar. & 6.23 & 1.28 & 9.63 & 0.64 & 27 & 6.84 & 6.83 & 6.82 \\
\hline & Apr. & 7.03 & 1.85 & 9.13 & 0.77 & 8 & 7.08 & 6.89 & 6.9 \\
\hline & May & 6.75 & 1.70 & 9.09 & 0.74 & 0 & 6.75 & 6.11 & 6.17 \\
\hline & Jun. & 5.53 & 1.31 & 8.42 & 0.65 & 0 & 5.53 & 4.82 & 4.87 \\
\hline & Jul. & 4 & 0.80 & 9.07 & 0.44 & 0 & 4.00 & 3.53 & 3.57 \\
\hline & Aug. & 3.8 & 0.77 & 9.35 & 0.40 & 1 & 3.80 & 3.59 & 3.62 \\
\hline & Sep. & 5.2 & 1.01 & 9.27 & 0.56 & 20 & 5.46 & 5.45 & 5.46 \\
\hline & Oct. & 5.32 & 1.01 & 9.51 & 0.55 & 38 & 6.45 & 6.28 & 6.25 \\
\hline & Nov. & 4.72 & 0.90 & 9.23 & 0.51 & 50 & 6.79 & 6.18 & 6.12 \\
\hline & Dec. & 4.57 & 0.38 & 8.96 & 0.51 & 56 & 7.67 & 6.55 & 6.47 \\
\hline & Average & 5.23 & 1.04 & 9.12 & 0.57 & 22 & 6.16 & 5.72 & 5.71 \\
\hline \multirow[t]{13}{*}{ Ranchi } & Jan. & 4.34 & 0.39 & 8.69 & 0.49 & 54 & 6.89 & 6.03 & 5.96 \\
\hline & Feb. & 4.91 & 0.96 & 9.07 & 0.54 & 42 & 6.29 & 6.00 & 5.96 \\
\hline & Mar. & 5.78 & 1.12 & 9.62 & 0.60 & 27 & 6.35 & 6.34 & 6.33 \\
\hline & Apr. & 6.16 & 1.39 & 9.12 & 0.67 & 8 & 6.21 & 6.05 & 6.07 \\
\hline & May & 5.88 & 1.28 & 9.08 & 0.64 & 0 & 5.88 & 5.31 & 5.36 \\
\hline & Jun. & 4.65 & 0.99 & 8.42 & 0.55 & 0 & 4.65 & 4.04 & 4.08 \\
\hline & Jul. & 4.02 & 0.80 & 9.06 & 0.44 & 0 & 4.02 & 3.55 & 3.59 \\
\hline & Aug. & 3.85 & 0.77 & 9.35 & 0.41 & 2 & 3.85 & 3.65 & 3.67 \\
\hline & Sep. & 4.13 & 0.80 & 9.26 & 0.44 & 20 & 4.34 & 4.34 & 4.34 \\
\hline & Oct. & 4.37 & 0.82 & 9.50 & 0.46 & 38 & 5.31 & 5.17 & 5.14 \\
\hline & Nov. & 4.26 & 0.82 & 9.22 & 0.46 & 50 & 6.12 & 5.57 & 5.52 \\
\hline & Dec. & 4.07 & 0.43 & 8.95 & 0.45 & 56 & 6.75 & 5.80 & 5.73 \\
\hline & Average & 4.7 & 0.88 & 9.11 & 0.51 & 22.09 & 5.56 & 5.15 & 5.15 \\
\hline
\end{tabular}


Table 2 continued

\begin{tabular}{|c|c|c|c|c|c|c|c|c|c|}
\hline Cities & Months & $H_{g}$ & $H_{d}$ & $H_{0}$ & $K_{t}$ & $\beta_{o p t}$ & $H_{T}$ & $\mathrm{HL}$ & HY \\
\hline \multirow[t]{13}{*}{ Shillong } & Jan. & 3.91 & 0.43 & 8.53 & 0.45 & 55 & 6.39 & 5.63 & 5.53 \\
\hline & Feb. & 4.63 & 0.91 & 8.92 & 0.51 & 44 & 6.08 & 5.80 & 5.75 \\
\hline & Mar. & 5.35 & 1.02 & 9.47 & 0.56 & 29 & 5.98 & 5.96 & 5.95 \\
\hline & Apr. & 5.86 & 1.30 & 8.97 & 0.65 & 10 & 5.94 & 5.78 & 5.81 \\
\hline & May & 5.11 & 1.04 & 8.92 & 0.57 & 0 & 5.11 & 4.6 & 4.65 \\
\hline & Jun. & 4.56 & 0.99 & 8.25 & 0.55 & 0 & 4.56 & 3.93 & 4.00 \\
\hline & Jul. & 4.46 & 0.88 & 8.90 & 0.50 & 0 & 4.46 & 3.91 & 3.97 \\
\hline & Aug. & 4.14 & 0.81 & 9.19 & 0.45 & 4 & 4.15 & 3.92 & 3.96 \\
\hline & Sep. & 3.89 & 0.78 & 9.11 & 0.42 & 22 & 4.13 & 4.13 & 4.13 \\
\hline & Oct. & 4.21 & 0.81 & 9.35 & 0.45 & 40 & 5.22 & 5.09 & 5.05 \\
\hline & Nov. & 4.34 & 0.41 & 9.06 & 0.47 & 53 & 6.79 & 6.09 & 5.99 \\
\hline & Dec. & 4.008 & 0.43 & 8.78 & 0.45 & 57 & 6.91 & 5.95 & 5.84 \\
\hline & Average & 4.54 & 0.82 & 8.959 & 0.50 & 23.54 & 5.48 & 5.07 & 5.05 \\
\hline \multirow[t]{13}{*}{ Varanasi } & Jan. & 3.58 & 0.47 & 8.52 & 0.42 & 55 & 5.79 & 5.12 & 5.03 \\
\hline & Feb. & 4.76 & 0.95 & 8.91 & 0.53 & 44 & 6.26 & 5.98 & 5.92 \\
\hline & Mar. & 5.81 & 1.16 & 9.46 & 0.61 & 29 & 6.48 & 6.47 & 6.46 \\
\hline & Apr. & 6.42 & 1.57 & 8.96 & 0.71 & 10 & 6.49 & 6.32 & 6.36 \\
\hline & May & 6.39 & 1.58 & 8.91 & 0.71 & 0 & 6.39 & 5.77 & 5.84 \\
\hline & Jun. & 5.79 & 1.51 & 8.24 & 0.70 & 0 & 5.79 & 5.03 & 5.11 \\
\hline & Jul. & 4.35 & 0.86 & 8.89 & 0.48 & 0 & 4.35 & 3.82 & 3.88 \\
\hline & Aug. & 4.8 & 0.92 & 9.18 & 0.52 & 4 & 4.81 & 4.55 & 4.59 \\
\hline & Sep. & 4.54 & 0.88 & 9.11 & 0.49 & 22 & 4.82 & 4.82 & 4.82 \\
\hline & Oct. & 4.76 & 0.90 & 9.34 & 0.51 & 40 & 5.92 & 5.76 & 5.72 \\
\hline & Nov. & 4.01 & 0.44 & 9.05 & 0.44 & 53 & 6.25 & 5.61 & 5.52 \\
\hline & Dec. & 3.37 & 0.52 & 8.77 & 0.38 & 57 & 5.68 & 4.93 & 4.84 \\
\hline & Average & 4.8 & 0.98 & 8.95 & 0.54 & 23.54 & 5.75 & 5.35 & 5.34 \\
\hline \multirow[t]{13}{*}{ Patna } & Jan. & 3.61 & 0.47 & 8.50 & 0.42 & 55 & 5.86 & 5.18 & 5.08 \\
\hline & Feb. & 4.72 & 0.94 & 8.90 & 0.53 & 44 & 6.22 & 5.94 & 5.87 \\
\hline & Mar. & 5.81 & 1.17 & 9.45 & 0.61 & 29 & 6.50 & 6.48 & 6.47 \\
\hline & Apr. & 6.35 & 1.53 & 8.95 & 0.70 & 10 & 6.42 & 6.25 & 6.29 \\
\hline & May & 6.29 & 1.53 & 8.90 & 0.70 & 0 & 6.29 & 5.67 & 5.75 \\
\hline & Jun. & 5.63 & 1.42 & 8.23 & 0.68 & 0 & 5.63 & 4.88 & 4.96 \\
\hline & Jul. & 4.36 & 0.87 & 8.87 & 0.49 & 0 & 4.36 & 3.83 & 3.89 \\
\hline & Aug. & 4.64 & 0.89 & 9.17 & 0.50 & 4 & 4.64 & 4.39 & 4.44 \\
\hline & Sep. & 4.55 & 0.88 & 9.09 & 0.50 & 22 & 4.84 & 4.83 & 4.84 \\
\hline & Oct. & 4.64 & 0.88 & 9.33 & 0.49 & 40 & 5.78 & 5.63 & 5.58 \\
\hline & Nov. & 4.08 & 0.44 & 9.04 & 0.45 & 53 & 6.38 & 5.73 & 5.63 \\
\hline & Dec. & 3.29 & 0.53 & 8.76 & 0.37 & 57 & 5.54 & 4.82 & 4.72 \\
\hline & Average & 4.83 & 0.96 & 8.93 & 0.54 & 23.54 & 5.70 & 5.32 & 5.30 \\
\hline \multirow[t]{13}{*}{ Jodhpur } & Jan. & 4.31 & 0.38 & 8.45 & 0.51 & 56 & 7.24 & 6.36 & 6.23 \\
\hline & Feb. & 5.05 & 1.04 & 8.85 & 0.57 & 45 & 6.69 & 6.40 & 6.32 \\
\hline & Mar. & 6.04 & 1.26 & 9.40 & 0.64 & 30 & 6.77 & 6.76 & 6.74 \\
\hline & Apr. & 6.73 & 1.76 & 8.91 & 0.75 & 11 & 6.82 & 6.63 & 6.68 \\
\hline & May & 6.97 & 1.94 & 8.85 & 0.78 & 0 & 6.97 & 6.30 & 6.39 \\
\hline & Jun. & 6.55 & 2.01 & 8.18 & 0.80 & 0 & 6.55 & 5.71 & 5.81 \\
\hline & Jul. & 5.46 & 1.18 & 8.82 & 0.61 & 0 & 5.46 & 4.79 & 4.88 \\
\hline & Aug. & 5.41 & 1.10 & 9.12 & 0.59 & 4 & 5.43 & 5.13 & 5.19 \\
\hline & Sep. & 5.85 & 1.28 & 9.05 & 0.64 & 22 & 6.22 & 6.21 & 6.22 \\
\hline & Oct. & 5.3 & 1.04 & 9.28 & 0.57 & 40 & 6.64 & 6.46 & 6.41 \\
\hline & Nov. & 4.49 & 0.39 & 8.99 & 0.49 & 54 & 7.17 & 6.42 & 6.30 \\
\hline & Dec. & 4.12 & 0.41 & 8.71 & 0.47 & 58 & 7.27 & 6.26 & 6.12 \\
\hline & Average & 5.52 & 1.15 & 8.88 & 0.62 & 24 & 6.60 & 6.12 & 6.11 \\
\hline
\end{tabular}


Table 2 continued

\begin{tabular}{|c|c|c|c|c|c|c|c|c|c|}
\hline Cities & Months & $H_{g}$ & $H_{d}$ & $H_{0}$ & $K_{t}$ & $\beta_{o p t}$ & $H_{T}$ & $\mathrm{HL}$ & HY \\
\hline \multirow[t]{13}{*}{ Lucknow } & Jan. & 4.44 & 0.36 & 8.43 & 0.52 & 56 & 7.53 & 6.61 & 6.48 \\
\hline & Feb. & 5.43 & 1.17 & 8.83 & 0.61 & 45 & 7.19 & 6.88 & 6.80 \\
\hline & Mar. & 5.97 & 1.24 & 9.38 & 0.63 & 30 & 6.70 & 6.69 & 6.68 \\
\hline & Apr. & 6.76 & 1.79 & 8.89 & 0.76 & 11 & 6.85 & 6.66 & 6.71 \\
\hline & May & 7.14 & 2.07 & 8.82 & 0.80 & 0 & 7.14 & 6.46 & 6.55 \\
\hline & Jun. & 6.06 & 1.70 & 8.16 & 0.74 & 0 & 6.06 & 5.26 & 5.35 \\
\hline & Jul. & 5.49 & 1.20 & 8.80 & 0.62 & 0 & 5.49 & 4.82 & 4.90 \\
\hline & Aug. & 5.32 & 1.08 & 9.10 & 0.58 & 5 & 5.34 & 5.05 & 5.10 \\
\hline & Sep. & 5.52 & 1.16 & 9.03 & 0.61 & 23 & 5.89 & 5.88 & 5.89 \\
\hline & Oct. & 5.63 & 1.15 & 9.26 & 0.60 & 41 & 7.05 & 6.87 & 6.82 \\
\hline & Nov. & 4.78 & 0.35 & 8.96 & 0.53 & 54 & 7.72 & 6.90 & 6.78 \\
\hline & Dec. & 4.19 & 0.40 & 8.68 & 0.48 & 59 & 7.47 & 6.42 & 6.29 \\
\hline & Average & 5.56 & 1.14 & 8.86 & 0.62 & 24.36 & 6.70 & 6.21 & 6.19 \\
\hline \multirow[t]{13}{*}{ Jaipur } & Jan. & 4.25 & 0.38 & 8.39 & 0.50 & 57 & 7.24 & 6.36 & 6.21 \\
\hline & Feb. & 5.01 & 1.03 & 8.79 & 0.56 & 45 & 6.69 & 6.40 & 6.31 \\
\hline & Mar. & 6.11 & 1.30 & 9.34 & 0.65 & 30 & 6.88 & 6.87 & 6.85 \\
\hline & Apr. & 7.08 & 2.01 & 8.85 & 0.80 & 11 & 7.18 & 6.98 & 7.04 \\
\hline & May & 7.25 & 2.17 & 8.78 & 0.82 & 0 & 7.25 & 6.56 & 6.67 \\
\hline & Jun. & 6.65 & 2.13 & 8.12 & 0.81 & 0 & 6.65 & 5.80 & 5.91 \\
\hline & Jul. & 5.13 & 1.08 & 8.75 & 0.58 & 0 & 5.13 & 4.49 & 4.59 \\
\hline & Aug. & 4.88 & 0.96 & 9.06 & 0.53 & 5 & 4.90 & 4.63 & 4.69 \\
\hline & Sep. & 5.45 & 1.14 & 8.99 & 0.60 & 23 & 5.83 & 5.82 & 5.83 \\
\hline & Oct. & 5.04 & 0.98 & 9.22 & 0.54 & 41 & 6.37 & 6.21 & 6.14 \\
\hline & Nov. & 4.27 & 0.41 & 8.92 & 0.47 & 54 & 6.89 & 6.19 & 6.05 \\
\hline & Dec. & 3.74 & 0.46 & 8.64 & 0.43 & 59 & 6.62 & 5.72 & 5.58 \\
\hline & Average & 5.4 & 1.17 & 8.82 & 0.61 & 24.36 & 6.47 & 6.92 & 5.99 \\
\hline \multirow[t]{13}{*}{ New Delhi } & Jan. & 3.7 & 0.44 & 8.26 & 0.44 & 58 & 6.39 & 5.65 & 5.52 \\
\hline & Feb. & 4.56 & 0.93 & 8.67 & 0.52 & 47 & 6.23 & 5.96 & 5.88 \\
\hline & Mar. & 5.73 & 1.19 & 9.22 & 0.62 & 32 & 6.54 & 6.53 & 6.51 \\
\hline & Apr. & 6.68 & 1.82 & 8.73 & 0.76 & 13 & 6.81 & 6.62 & 6.67 \\
\hline & May & 6.78 & 1.92 & 8.65 & 0.78 & 0 & 6.78 & 6.12 & 6.22 \\
\hline & Jun. & 6.26 & 1.92 & 7.99 & 0.78 & 0 & 6.26 & 5.43 & 5.54 \\
\hline & Jul. & 5.29 & 1.17 & 8.62 & 0.61 & 0 & 5.29 & 4.63 & 4.72 \\
\hline & Aug. & 4.94 & 0.99 & 8.93 & 0.55 & 8 & 4.96 & 4.69 & 4.75 \\
\hline & Sep. & 5.25 & 1.09 & 8.87 & 0.59 & 26 & 5.67 & 5.66 & 5.67 \\
\hline & Oct. & 4.66 & 0.90 & 9.10 & 0.51 & 43 & 6.00 & 5.84 & 5.78 \\
\hline & Nov. & 3.92 & 0.44 & 8.79 & 0.44 & 55 & 6.45 & 5.81 & 5.68 \\
\hline & Dec. & 3.31 & 0.51 & 8.50 & 0.38 & 60 & 5.93 & 5.17 & 5.04 \\
\hline & Average & 5.09 & 1.11 & 8.69 & 0.58 & 25.81 & 6.11 & 5.68 & 5.67 \\
\hline \multirow[t]{13}{*}{ Dehradun } & Jan. & 3.58 & 0.44 & 8.08 & 0.44 & 59 & 6.44 & 5.71 & 5.52 \\
\hline & Feb. & 4.4 & 0.91 & 8.51 & 0.51 & 48 & 6.18 & 5.91 & 5.80 \\
\hline & Mar. & 5.47 & 1.13 & 9.05 & 0.60 & 34 & 6.35 & 6.34 & 6.31 \\
\hline & Apr. & 6.35 & 1.69 & 8.57 & 0.74 & 15 & 6.51 & 6.33 & 6.40 \\
\hline & May & 6.95 & 2.14 & 8.47 & 0.82 & 0 & 6.95 & 6.27 & 6.41 \\
\hline & Jun. & 6.06 & 1.88 & 7.81 & 0.77 & 0 & 6.06 & 5.23 & 5.38 \\
\hline & Jul. & 5.25 & 1.19 & 8.43 & 0.62 & 0 & 5.25 & 4.58 & 4.71 \\
\hline & Aug. & 4.8 & 0.98 & 8.76 & 0.54 & 8 & 4.84 & 4.58 & 4.66 \\
\hline & Sep. & 5.32 & 1.15 & 8.72 & 0.61 & 26 & 5.81 & 5.80 & 5.81 \\
\hline & Oct. & 5.13 & 1.05 & 8.93 & 0.57 & 44 & 6.74 & 6.57 & 6.47 \\
\hline & Nov. & 4.22 & 0.40 & 8.60 & 0.49 & 57 & 7.29 & 6.56 & 6.36 \\
\hline & Dec. & 3.53 & 0.47 & 8.31 & 0.42 & 61 & 6.70 & 5.83 & 5.62 \\
\hline & Average & 5.09 & 1.12 & 8.52 & 0.59 & 26.63 & 6.26 & 5.81 & 5.79 \\
\hline
\end{tabular}


Table 2 continued

\begin{tabular}{|c|c|c|c|c|c|c|c|c|c|}
\hline Cities & Months & $H_{g}$ & $H_{d}$ & $H_{0}$ & $K_{t}$ & $\beta_{o p t}$ & $H_{T}$ & $\mathrm{HL}$ & HY \\
\hline \multirow[t]{13}{*}{ Hamirpur } & Jan. & 2.43 & 0.63 & 7.93 & 0.30 & 57 & 4.13 & 3.75 & 3.65 \\
\hline & Feb. & 2.87 & 0.75 & 8.36 & 0.34 & 50 & 4.02 & 3.87 & 3.80 \\
\hline & Mar. & 4.79 & 0.96 & 8.91 & 0.53 & 36 & 5.66 & 5.65 & 5.63 \\
\hline & Apr. & 5.22 & 1.18 & 8.43 & 0.61 & 17 & 5.39 & 5.24 & 5.30 \\
\hline & May & 6.14 & 1.67 & 8.32 & 0.73 & 0 & 6.14 & 5.52 & 5.64 \\
\hline & Jun. & 4.95 & 1.28 & 7.65 & 0.64 & 0 & 4.95 & 4.23 & 4.35 \\
\hline & Jul. & 4.06 & 0.86 & 8.27 & 0.49 & 0 & 4.06 & 3.53 & 3.63 \\
\hline & Aug. & 3.48 & 0.76 & 8.61 & 0.40 & 11 & 3.52 & 3.33 & 3.38 \\
\hline & Sep. & 3.98 & 0.82 & 8.58 & 0.46 & 29 & 4.40 & 4.39 & 4.40 \\
\hline & Oct. & 4.21 & 0.85 & 8.78 & 0.47 & 47 & 5.66 & 5.52 & 5.44 \\
\hline & Nov. & 3.16 & 0.53 & 8.45 & 0.37 & 59 & 5.40 & 4.91 & 4.78 \\
\hline & Dec. & 2.85 & 0.56 & 8.15 & 0.35 & 63 & 5.37 & 4.73 & 4.58 \\
\hline & Average & 4.01 & 0.90 & 8.37 & 0.47 & 28.36 & 4.89 & 4.56 & 4.55 \\
\hline \multirow[t]{13}{*}{ Srinagar } & Jan. & 1.32 & 0.89 & 7.67 & 0.17 & 52 & 1.67 & 1.64 & 1.62 \\
\hline & Feb. & 2.71 & 0.58 & 8.12 & 0.33 & 48 & 4.05 & 3.88 & 3.79 \\
\hline & Mar. & 3.95 & 0.81 & 8.67 & 0.45 & 39 & 4.78 & 4.78 & 4.75 \\
\hline & Apr. & 5.06 & 1.18 & 8.20 & 0.61 & 20 & 5.28 & 5.13 & 5.20 \\
\hline & May & 5.62 & 1.48 & 8.06 & 0.69 & 3 & 5.62 & 5.05 & 5.19 \\
\hline & Jun. & 6.18 & 2.24 & 7.39 & 0.83 & 0 & 6.18 & 5.33 & 5.51 \\
\hline & Jul. & 5.6 & 1.49 & 8.00 & 0.69 & 0 & 5.60 & 4.88 & 5.04 \\
\hline & Aug. & 5.2 & 1.19 & 8.37 & 0.62 & 13 & 5.29 & 5.00 & 5.10 \\
\hline & Sep. & 5.06 & 1.14 & 8.35 & 0.60 & 31 & 5.68 & 5.67 & 5.68 \\
\hline & Oct. & 3.85 & 0.81 & 8.54 & 0.45 & 49 & 5.35 & 5.22 & 5.13 \\
\hline & Nov. & 2.56 & 0.62 & 8.18 & 0.31 & 59 & 4.40 & 4.05 & 3.92 \\
\hline & Dec. & 1.94 & 0.73 & 7.87 & 0.24 & 61 & 3.36 & 3.07 & 2.96 \\
\hline & Average & 4.09 & 1.10 & 8.12 & 0.50 & 29.72 & 4.77 & 4.48 & 4.49 \\
\hline
\end{tabular}

June, July and August) and $49^{\circ}$ (December) for Hyderabad, $0^{\circ}$ (May, June, July and August) and $49^{\circ}$ (December) for Vishakapatnam, $0^{\circ}$ (May, June, July and August) and $50^{\circ}$ (December) for Pune, $0^{\circ}$ (May, June, July and August) and $51^{\circ}$ (December) for Mumbai, $0^{\circ}$ (May, June, July and August) and $54^{\circ}$ (December) for Nagpur, $0^{\circ}$ (May, June, July and August) and $55^{\circ}$ (December) for Bhavnagar, $0^{\circ}$
(May, June and July) and $54^{\circ}$ (December) for Kolkatta, $0^{\circ}$ (May, June and July) and $57^{\circ}$ (December) for Ahmedabad, $0^{\circ}$ (May, June and July) and $56^{\circ}$ (December) for Bhopal, $0^{\circ}$ (May, June and July) and $56^{\circ}$ (December) for Ranchi, $0^{\circ}$ (May, June and July) and $57^{\circ}$ (December) for Shillong, $0^{\circ}$ (May, June and July) and $57^{\circ}$ (December) for Varanasi, $0^{\circ}$ (May, June and July) and $57^{\circ}$ (December) for Patna, $0^{\circ}$

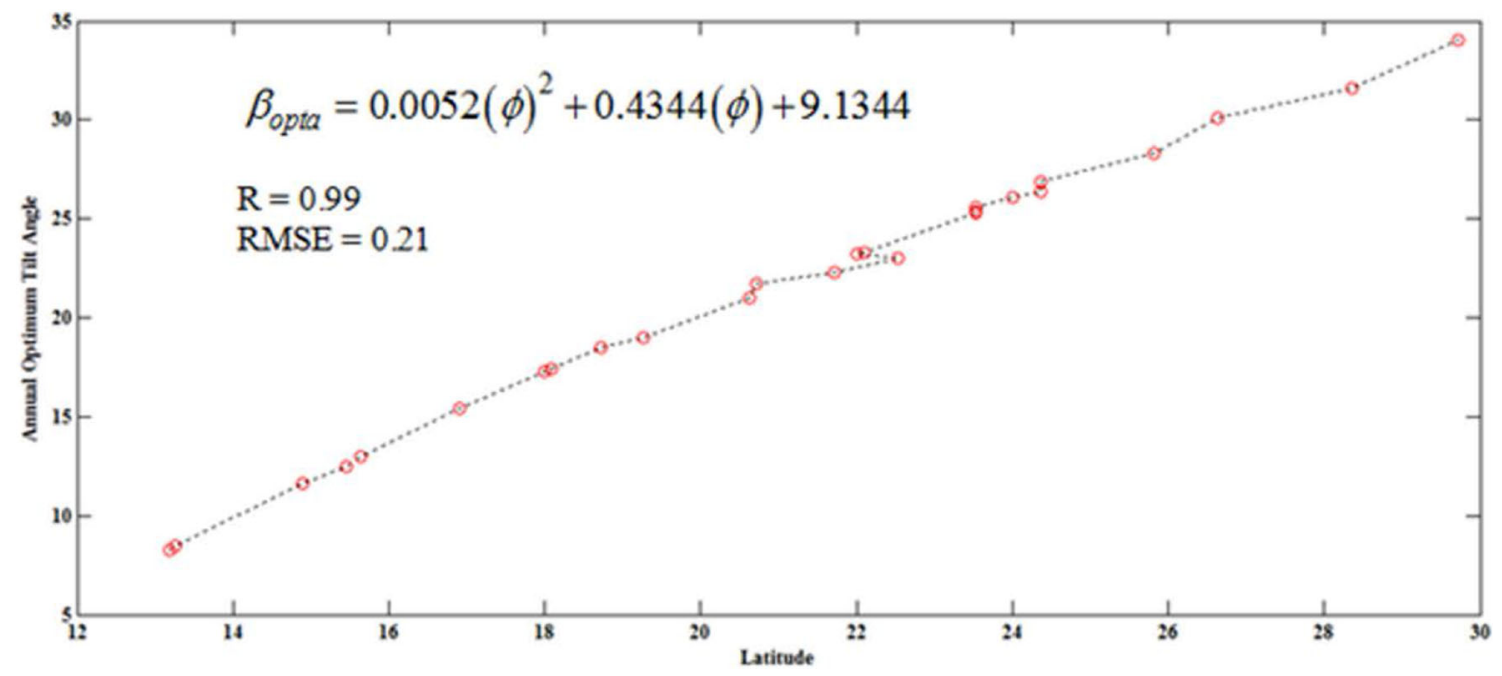

Figure 3. Variation of average annual OPTA with latitude. 
(May, June and July) and $58^{\circ}$ (December) for Jodhpur, $0^{\circ}$ (May, June and July) and $59^{\circ}$ (December) for Lucknow, $0^{\circ}$ (May, June and July) and $59^{\circ}$ (December) for Jaipur, $0^{\circ}$ (May, June and July) and $60^{\circ}$ (December) for New Delhi, $0^{\circ}$ (May, June and July) and $61^{\circ}$ (December) for Dehradun, $0^{\circ}$ (May, June and July) and $63^{\circ}$ (December) for Hamirpur, $0^{\circ}$ (June and July) and $61^{\circ}$ (December) for Srinagar. The variation of average annual OPTA $\left(\beta_{\text {opta }}\right)$ with latitude is shown in figure 3.

The percentage increase in maximum SR at monthly optimum tilt angle in comparison to annual and latitude based tilt angles are shown in table 3 . The increase in maximum SR at monthly OPTA in comparison to latitude based tilt angle and annual OPTA varies from $5.85 \%$ to $8.08 \%$ and $5.95 \%$ to $8.34 \%$, respectively, showing monthly OPTA is beneficial for maximum power generation for different cities in India.

Additionally, the correlation equations of the monthly OPTA $\left(\beta_{\text {opt }}\right)$ based on the extraterrestrial SR $\left(H_{0}\right)$ are developed for different months for India. These correlation equations are given below

The accuracy of correlations equations are evaluated by using the most widely used statistical indicators which are Mean Bias Error (MBE), Root Mean Square Error (RMSE),

Table 3. Increase in SR at monthly OPTA in comparison to latitude and annual optimum based tilt angle.

\begin{tabular}{lcc}
\hline & $\begin{array}{c}\text { \% increase in } \\
\text { comparison to } \\
\text { latitude }\end{array}$ & $\begin{array}{c}\text { \% increase in } \\
\text { comparison to annual } \\
\text { CPTA }\end{array}$ \\
\hline Minicoy & 6.05 & 5.96 \\
Thiruvanathpuram & 5.85 & 5.95 \\
Port Blair & 6.43 & 6.25 \\
Bangalore & 6.40 & 6.40 \\
Chennai & 6.26 & 6.35 \\
Panjim & 6.63 & 6.53 \\
Hyderabad & 6.83 & 6.80 \\
Vishakapatnam & 6.91 & 6.87 \\
Pune & 6.85 & 6.84 \\
Mumbai & 7.002 & 6.982 \\
Nagpur & 7.266 & 7.305 \\
Bhavnagar & 7.450 & 7.572 \\
Kolkatta & 7.526 & 7.571 \\
Ahmedabad & 7.615 & 7.671 \\
Bhopal & 7.560 & 7.711 \\
Ranchi & 7.793 & 7.940 \\
Shillong & 8.086 & 8.343 \\
Varanasi & 7.436 & 7.577 \\
Patna & 7.385 & 7.547 \\
Jodhpur & 7.772 & 7.984 \\
Lucknow & 7.821 & 8.081 \\
Jaipur & 7.707 & 7.959 \\
New Delhi & 7.570 & 7.760 \\
Dehradun & 7.689 & 8.080 \\
Hamirpur & 7.213 & 7.378 \\
Srinagar & 6.473 & 6.117 \\
\hline & & \\
\hline
\end{tabular}

$$
\begin{aligned}
& \beta_{\text {opt }}=-10.5686\left(H_{o}\right)^{2}+172.3649\left(H_{o}\right)-646.0214 \quad \text { for January } \\
& \beta_{\text {opt }}=-7.0820\left(H_{o}\right)^{2}+115.0357\left(H_{o}\right)-418.2813 \quad \text { for February } \\
& \beta_{o p t}=-4.2248\left(H_{o}\right)^{2}+65.8137\left(H_{o}\right)-215.0140 \quad \text { for March }(6) \\
& \beta_{\text {opt }}=0.7043\left(H_{o}\right)^{2}-25.8401\left(H_{o}\right)+184.9276 \quad \text { for April (7) } \\
& \beta_{\text {opt }}=\left(0.0003\left(H_{o}\right)^{4}-0.0128\left(H_{o}\right)^{3}+0.1753\left(H_{o}\right)^{2} \quad \text { for May }(8)\right. \\
& \left.-1.0691\left(H_{o}\right)+2.4426\right) \times 10^{4} \\
& \beta_{\text {opt }}=0 \\
& \beta_{\text {opt }}=0 \\
& \beta_{\text {opt }}=5.2105\left(H_{o}\right)^{2}-104.6942\left(H_{o}\right)+525.5833 \\
& \beta_{\text {opt }}=-5.3033\left(H_{o}\right)^{2}+82.3239\left(H_{o}\right)-287.4305 \\
& \beta_{\text {opt }}=-4.3097\left(H_{o}\right)^{2}+67.1274\left(H_{o}\right)-210.7192 \\
& \beta_{\text {opt }}=-4.6538\left(H_{o}\right)^{2}+72.3703\left(H_{o}\right)-221.0626 \\
& \beta_{\text {opt }}=-5.9455\left(H_{o}\right)^{2}+94.5274\left(H_{o}\right)-313.9101
\end{aligned}
$$

$\mathrm{t}$-statistic (t-stat) and correlation coefficient (R) (table 4). The MBE, RMSE, $t$-stat and $r$ are given by following equations.

$$
\begin{gathered}
\mathrm{MBE}=\frac{1}{n} \sum_{i=1}^{n}\left(y_{i}-x_{i}\right) \\
\mathrm{RMSE}=\left[\frac{1}{n} \sum_{i=1}^{n}\left(y_{i}-x_{i}\right)^{2}\right]^{1 / 2} \\
\mathrm{t}-\mathrm{stat}=\left(\frac{(n-1) \mathrm{MBE}^{2}}{\mathrm{RMSE}^{2}-\mathrm{MBE}^{2}}\right)^{1 / 2} \\
\mathrm{R}=\left[\frac{\left(Z_{t}-Z_{r}\right)}{Z_{t}}\right]^{1 / 2}
\end{gathered}
$$

Table 4. Error evaluation of obtained correlation equations.

\begin{tabular}{crccc}
\hline Equation no. & MBE & RMSE & t -statistic & R \\
\hline$(13)$ & $1.92 \times 10^{-13}$ & 0.9771 & $6.53 \times 10^{-13}$ & 0.98 \\
$(14)$ & $-7.21 \times 10^{-14}$ & 0.5496 & $4.35 \times 10^{-15}$ & 0.99 \\
$(15)$ & $2.04 \times 10^{-13}$ & 0.6503 & $1.04 \times 10^{-12}$ & 0.98 \\
$(16)$ & $-2.62 \times 10^{-14}$ & 0.7544 & $1.15 \times 10^{-13}$ & 0.91 \\
$(17)$ & $-9.04 \times 10^{-12}$ & 0.1256 & $2.40 \times 10^{-10}$ & 0.97 \\
$(20)$ & $3.49 \times 10^{-14}$ & 0.7036 & $1.64 \times 10^{-13}$ & 0.98 \\
$(21)$ & $-4.37 \times 10^{-15}$ & 0.6700 & $2.16 \times 10^{-14}$ & 0.99 \\
$(22)$ & $-6.77 \times 10^{-14}$ & 0.6062 & $3.70 \times 10^{-13}$ & 0.99 \\
$(23)$ & $-9.40 \times 10^{-14}$ & 0.4229 & $7.37 \times 10^{-13}$ & 0.99 \\
$(24)$ & $2.90 \times 10^{-13}$ & 0.6421 & $1.50 \times 10^{-12}$ & 0.99 \\
\hline
\end{tabular}




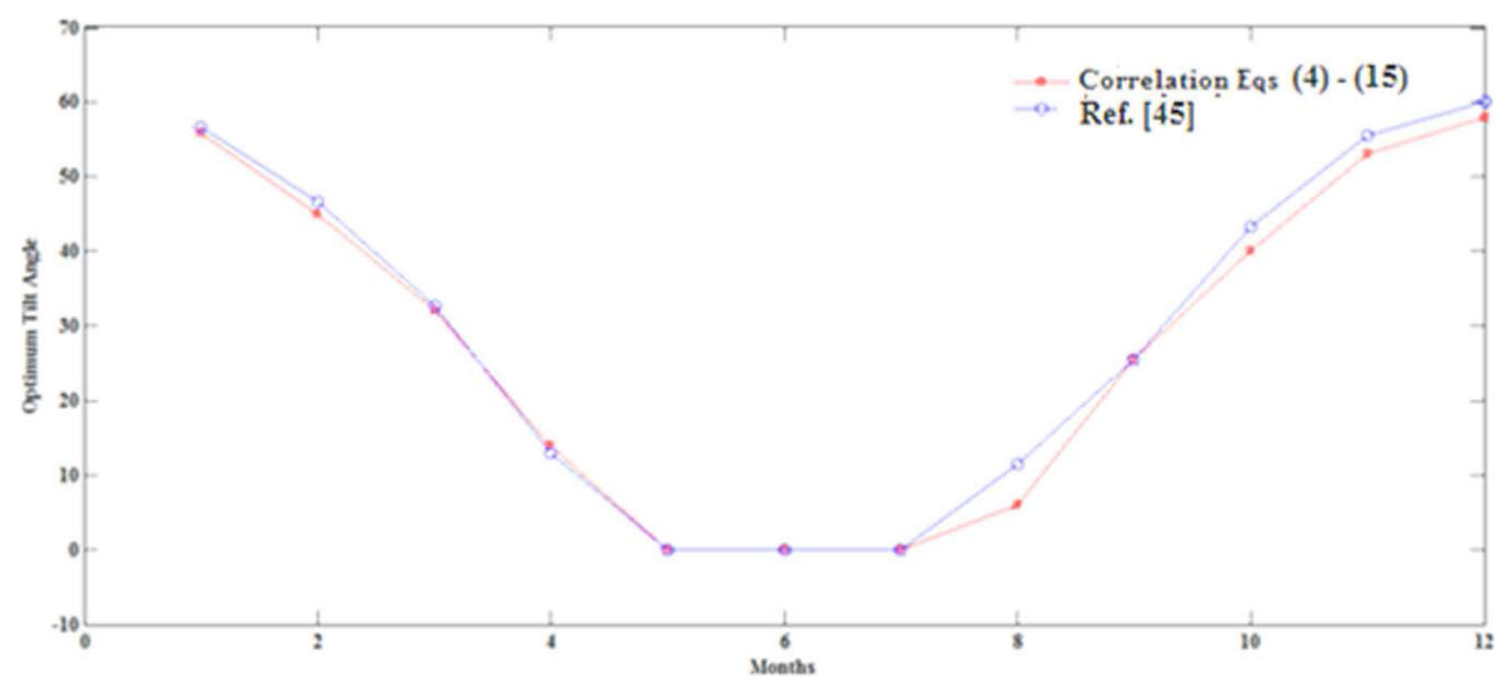

Figure 4. Comparison of the results obtained in the literature for the city of New Delhi.

Table 5. Comparisons of OPTA calculation with PV Syst.

\begin{tabular}{lll}
\hline Cities & OPTA and maximum SR obtained in this study & OPTA and maximum SR given by PV Syst \\
\hline Hamirpur & OPTA is $28.36^{\circ}$ and maximum SR is $4.56 \mathrm{kWh} / \mathrm{m}^{2} /$ day & OPTA is $28^{\circ}$ and maximum SR is $4.408 \mathrm{kWh} / \mathrm{m}^{2} /$ day \\
New Delhi & OPTA is $25.81^{\circ}$ and maximum SR is $5.68 \mathrm{kWh} / \mathrm{m}^{2} /$ day & OPTA is $27^{\circ}$ and maximum SR is $5.567 \mathrm{kWh} / \mathrm{m}^{2} /$ day \\
\hline
\end{tabular}

$$
\begin{gathered}
Z_{t}=\sum_{i=1}^{n}\left(x_{o}-x_{i}\right)^{2} \\
Z_{r}=\sum_{i=1}^{n}\left(x_{i}-y_{i}\right)^{2} \\
x_{o}=\frac{1}{n} \sum_{i=1}^{n} x_{i}
\end{gathered}
$$

where $x_{i}$ is $\mathrm{i}^{\text {th }}$ optimum tilt angle, $y_{i}$ is $\mathrm{i}^{\text {th }}$ OPTA calculated by correlation equations.

The correlations equations 13 to 24 are used to find OPTA of New Delhi and is validated with results from previously published literature [45] shown in figure 4.

\subsection{Comparison of optimum tilt angle calculation with PV Syst}

The OPTA and maximum SR on tilted surface for Hamirpur and New Delhi cities are compared with PV Syst software are shown in table 5.

\section{Conclusions}

In this study optimum tilt angle $\left(\beta_{\text {opt }}\right)$ of 26 different Indian cities are calculated by changing tilt angle $(\beta)$ from $0^{\circ}$ to $90^{\circ}$ at step of $1^{\circ}$. The correlations of monthly optimum tilt angles are developed in terms of extraterrestrial solar radiation. Based on the results of study, the following conclusions are drawn.

1. It was found that the optimum tilt angle changed between $0^{\circ}$ (May, June, July) and 63० (December) throughout the year in India. The optimum tilt angle is maximum in December for all cities.

2. The months in which $\beta_{\text {opt }}$ is $0^{\circ}$ for different locations in India are April, May, June, July and August $\left(8.28^{\circ} \mathrm{N} \leq \phi \leq 15.4^{\circ} \mathrm{N}\right)$; May, June, July and August $\left(17.3^{\circ} \mathrm{N} \leq \phi \leq 21.7^{\circ} \mathrm{N}\right) ; \quad$ May, June and July $\left(22.3^{\circ} \mathrm{N} \leq \phi \leq 31.6^{\circ} \mathrm{N}\right)$; June and July $\left(\phi=34^{\circ} \mathrm{N}\right)$.

3. The average global solar radiation on monthly optimum tilted surface varies from 4.77 to $6.7 \mathrm{kWh} / \mathrm{m}^{2} /$ day throughout India.

4. The developed correlation Eqs. (4) to (15) in this study can be used to estimate the optimum tilt angle of a solar photovoltaic system in India. The root mean square error in correlation equations varies from 0.1256 to 0.9771 .

5. The annual average optimum tilts angle increase with latitude (figure 3). The RMSE in correlation of annual optimum tilt angle is $0.21^{\circ}$.

6. The increase in maximum solar radiation at monthly optimum tilt angle in comparison to latitude based tilt angle and annual optimum tilt angle varies from $5.85 \%$ to $8.08 \%$ and $5.95 \%$ to $8.34 \%$, respectively, showing monthly optimum tilt angle is beneficial for maximum power generation for different cities in India. 
Future research work is focused on experimental analysis of maximum solar radiation on optimum tilt angles for different cites in India.

\section{List of symbols}

HL Maximum solar radiation on latitude based tilt angle

HY Maximum solar radiation on annual optimum based tilt angle

$H_{d} \quad$ Diffused radiation on horizontal surface $\left(\mathrm{kWh} \mathrm{m}^{-2}\right.$ day $^{-1}$ )

$H_{g} \quad$ Global solar radiation on horizontal surface $\left(\mathrm{kWh} \mathrm{m}^{-2}\right.$ day $^{-1}$ )

$H_{0} \quad$ Extraterrestrial solar radiation $\left(\mathrm{kWh} \mathrm{m}^{-2}\right.$ day $\left.^{-1}\right)$

$H_{r} \quad$ Reflected radiation on horizontal surface $\left(\mathrm{kWh} \mathrm{m}^{-2}\right.$ day $^{-1}$ )

$H_{T} \quad$ Global solar radiation on tilted surface $\left(\mathrm{kWh} \mathrm{m}^{-2}\right.$ day $^{-1}$ )

$K_{t} \quad$ Monthly Average Clearness index

$R_{b} \quad$ Ratio of beam radiation on tilted surface to that on horizontal surface

\section{Greek symbols}

$\beta \quad$ Tilt angle $\left({ }^{\circ}\right)$

$\beta_{\text {opt }} \quad$ Monthly optimum tilt angle for maximizing incident solar radiation

$\beta_{\text {opta }} \quad$ Annual optimum tilt angle

$\delta \quad$ Declination angle $\left({ }^{\circ}\right)$

$\phi \quad$ Latitude of a place $\left({ }^{\circ}\right)$

$\rho \quad$ Reflectivity of ground (0.02)

$w \quad$ Hour angle $\left({ }^{\circ}\right)$

$w_{s s} \quad$ Sunset hour angle at tilted surface $\left({ }^{\circ}\right)$

\section{References}

[1] Ramachandra T V, Jain R and Krishnadas G 2011 Hotspots of solar potential in India. Renew. Sustain. Energy Rev. 15: 3178-3186

[2] https://en.wikipedia.org/wiki/Jawaharlal_Nehru_National_ Solar_Mission

[3] Yadav A K and Malik H 2015 Optimization of tilt angle for installation of solar photovoltaic system for six sites in India. In: International Conference on Energy Economics and Environment (ICEEE), Noida, India. March 27-28, pp. 1-4

[4] Ansari M F, Kharb R K, Luthra S, Shimmi S L and Chatterji S 2013 Analysis of barriers to implement solar power installations in India using interpretive structural modeling technique. Renew. Sustain. Energy Rev. 27: 163-174

[5] Khatib T, Mohamed A and Sopian K 2013 A review of photovoltaic systems size optimization techniques. Renew. Sustain. Energy Rev. 22: 454-465

[6] Yadav A K and Chandel S S 2015 Solar energy potential assessment of western Himalayan Indian state of Himachal Pradesh using J48 algorithm of WEKA in ANN based prediction model. Renew. Energy 75: 675-693
[7] Yadav A K, Malik H and Chandel S S 2014 Selection of most relevant input parameters using WEKA for artificial neural network based solar radiation prediction models. Renew. Sustain. Energy Rev. 31: 509-519

[8] Thakur V and Chandel S S 2013 Maximizing the solar gain of a grid-interactive solar photovoltaic power plant. Energy Technol. 1: 1-8

[9] Yadav P and Chandel S S 2014 Comparative analysis of diffused solar radiation models for optimum tilt angle determination for Indian locations. Appl. Solar Energy 50(1): 53-59

[10] Yadav A K Malik H and Chandel S S 2016 Tilt angle calculation for installation of PV systems for mountainous regions of Himachal Pradesh India. In: International Conference on Electrical Power and Energy Systems (ICEPES) Maulana Azad National Institute of Technology, Bhopal, India. Dec 14-16, pp. 205-209

[11] Agarwal A, Vashishtha V K and Mishra S N 2012 Comparative approach for the optimization of tilt angle to receive maximum radiation. Int. J. Eng. Res. Technol. 1(5): 1-9.

[12] Ulgen K 2006 Optimum tilt angle for solar collectors. Energy Sources A 28: 1171-1180

[13] Talebizadeh P, Mehrabian M Aand Abdolzadeh M 2011 Prediction of the optimum slope and surface azimuth angles using the Genetic Algorithm. Energy Build. 43: 2998-3005

[14] Pour H S S, Beheshti H K and Rahnama M 2011 The gain of the energy under the optimum angles of solar panels during a year in Isfahan, Iran. Energy Sources A. 33: 1281-1290

[15] Kacira M, Simsek M, Babur Y and Demirkol S 2004 Determining optimum tilt angles and orientations of photovoltaic panels in Sanliurfa, Turkey. Renew. Energy 29: 1265-1275

[16] Hartley L E, Martínwz-Lozano J A, Utrillas M P, Tena F and Pedrós R 1999 The optimisation of the angle of inclination of a solar collector to maximise the incident solar radiation. Renew. Energy 17: 291-309

[17] Mehleri E D, Zervas P L, Sarimveis H, Palyvos J A and Markatos N C 2010 Determination of the optimal tilt angle and orientation for solar photovoltaic arrays. Renew. Energy 35: $2468-2475$

[18] Siraki A G and Pillay P 2012 Study of optimum tilts angles for solar panels in different latitudes for urban applications. Sol. Energy 86: 1920-1928

[19] Chang Y 2010 Optimal the tilt angles for photovoltaic modules in Taiwan. Electr. Power Energy Syst. 32: 956-964

[20] Chatterjee A and Keyhani A 2012 Neural Network estimation of micro grid maximum solar power. IEEE Trans. Smart Grid 3(4): 1860-1866

[21] Khorasanizadeh H, Mohammadi K and Mostafaeipour A 2014 Establishing a diffuse solar radiation model for determining the optimum tilt angle of solar surfaces in Tabass, Iran. Energy Convers. Manag. 78: 805-814

[22] Bakirci K 2012 General models for optimum tilt angles of solar panels: Turkey case study. Renew. Sustain. Energy Rev. 16: 6149-6159

[23] Abdallah S 2004 The effect of using sun tracking systems on the voltage-current characteristics and power generation of flat plate photovoltaics. Energy Convers. Manag. 45: 1671-1679

[24] Beringer S, Schilke H, Lohse I and Seckmeyer G 2011 Case study showing that the tilt angle of photovoltaic plants is nearly irrelevant. Sol. Energy 85: 470-476 
[25] Bojić M, Bigot D, Miranville F, Parvedy-Patou A and Radulović J 2012 Optimizing performances of photovoltaic in Reunion Island-tilt angle. Prog. Photovolt. Res. Appl. 20: 923-935

[26] Chang Y 2009 Optimal design of discrete-value tilt angle of PV using sequential neural-network approximation and orthogonal array. Expert Syst. Appl. 36: 6010-6018

[27] Roux W G L 2016 Optimum tilt and azimuth angles for fixed solar collectors in South Africa using measured data. Renew. Energy 96: 603-612

[28] Heywood H 1971 Operating experience with solar water heating. IHVE J. 39: 63-69

[29] Lunde P J 1980 Solar Thermal Engineering: Space Heating and Hot Water Systems. New York: Wiley

[30] Chinnery D N W 1971 Solar water heating in South Africa. CSIR Rep. 248: 44

[31] Kern J and Harris I 1975 On the optimum tilt of a solar collector. Sol. Energy 17: 97-102

[32] Yellott H 1973 Utilization of sun and sky radiation for heating cooling of buildings. ASHRAE J. 15: 31-42

[33] Elminir H K, Ghitas A E, El-Hussainy F, Hamid R, Beheary M M and Abdel-Moneim K M 2006 Optimum solar flat plate collector slope: case study for Helwan, Egypt. Energy Convers. Manag. 47: 624-637

[34] Tang R, Wu T 2004 Optimal tilt-angles for solar collectors used in China. Appl. Energy 79: 239-248

[35] Talebizadeh P, Mehrabian M A and Abdolzadeh M 2011 Determination of optimum slope angles of solar collectors based on new correlations. Energy Sources A 33: 1567-1580
[36] Yadav A K and Chandel S S 2013 Tilt angle optimization to maximize incident solar radiation: a review. Renew. Sustain. Energy Rev. 23: 503-513

[37] Benghanem M 2011 Optimization of tilt angle for solar panel: case study for Madinah, Saudi Arabia. Appl. Energy 88: $1427-1433$

[38] https://teachengineeringprod.blob.core.windows.net/content/ cub_/lessons/cub_pveff/Images/cub_pveff_lesson01_figur e3web.jpg

[39] Spencer J W 1971 Fourier Series Representation on position of the sun. Search. 2(5): 172

[40] Copper P I 1969 The absorption of solar radiation in solar stills. Sol. Energy. 12(3): 333-346

[41] Liu B Y H and Jordan R C 1963 The long term average performance of flat plate solar energy collectors. Sol. Energy 7: 53

[42] Duffie J A and Beckman W 1980 Solar Engineering of Thermal Processes. New York: Wiley

[43] Collares-Pereira M and Rabl A 1979 The average distribution of solar radiation correlations between diffuse and hemispherical and between daily and hourly insolation values. Sol. Energy 22: 155

[44] Liu B Y H and Jordan R C 1962 Daily insolation on surfaces tilted toward the equator. ASHRAE Trans. 67: 526-541

[45] Ahmad M J and Tiwari G N 2009 Optimization of tilt angle for solar collector to receive maximum radiation. Open Renew. Energy J. 2: 19-24 